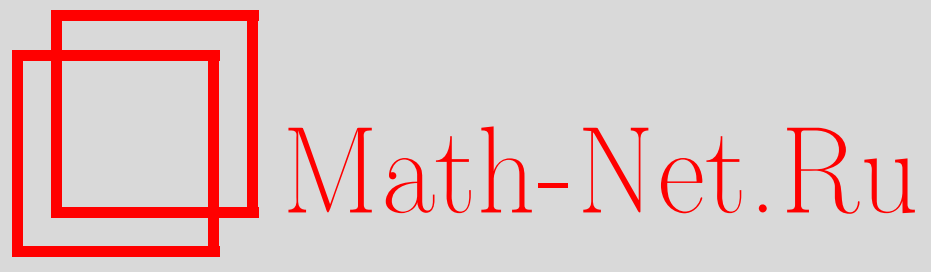

М. П. Фатеев, Метод когерентного потенциала для задачи диффузии на случайной решетке замещения, $T M \Phi$, 2006, том 149, номер 2, 252-261

DOI: https://doi.org/10.4213/tmf4232

Использование Общероссийского математического портала Math-Net.Ru подразумевает, что вы прочитали и согласны с пользовательским соглашением http://www . mathnet.ru/rus/agreement

Параметры загрузки:

IP: 3.82 .47 .9

26 апреля 2023 г., $07: 57: 55$

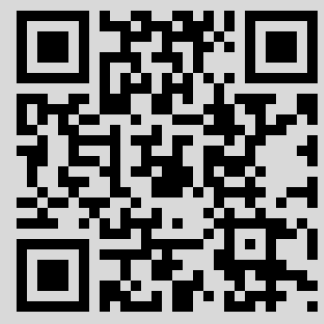




\title{
МЕТОД КОГЕРЕНТНОГО ПОТЕНЦИАЛА ДЛЯ ЗАДАЧИ ДИФФУЗИИ НА СЛУЧАЙНОЙ РЕШЕТКЕ ЗАМЕЩЕНИЯ
}

\begin{abstract}
На основе уравнения баланса рассмотрена задача диффузии на гиперрешетке со случайно распределенными недоступными узлами. С использованием диаграммных методов найдено самосогласованное выражение для конфигурационно-усредненной функции Грина в приближении когерентного потенциала. Показано, что указанный подход справедлив в широком диапазоне изменения концентраций доступных узлов. С помощью рассмотренного приближения найдена точная асимптотика для статического коэффициента диффузии при малой концентрации заблокированных узлов. Это позволяет с хорошей точностью оценивать пороги протекания для задачи диффузии со случайными узлами на произвольных гиперрешетках.
\end{abstract}

Ключевые слова: метод когерентного потенциала, функция Грина, диффузия, протекание, задача случайных узлов, диаграммные методы.

\section{1. ВВЕДЕНИЕ}

В последнее время возрос интерес к исследованию процессов переноса в неупорядоченных системах [1]. Задачи такого типа возникают при изучении прыжковой проводимости в примесных полупроводниках, переноса электронных возбуждений в органических соединениях, диффузии в сплавах и стеклах и т.д. Было показано, что в этом случае диффузия или прыжковая проводимость проявляют немарковское поведение [2], [3].

При исследовании процессов переноса на решетке со статическим беспорядком обычно рассматриваются две основные модели: задача случайных барьеров (связей) (ЗСБ) и задача случайных узлов (ЗСУ). В первом случае частота переходов $W_{n n^{\prime}}=W_{n^{\prime} n}$ зависит от состояния связи между узлами $n$ и $n^{\prime}$, по которым происходит переход. Во втором случае частота переходов $W_{n n^{\prime}}=W_{n}$ определяется состоянием узла $n$, на который происходит прыжок (здесь мы не рассматриваем модель ловушек, где частота переходов определяется состоянием узла, из которого происходит прыжок). При этом обычно предполагается отсутствие корреляций в

*Национальный научный центр "Харьковский физико-технический институт", Харьков, Украина. E-mail: mfateev@kipt.kharkov.ua 
распределении случайных узлов или связей. ЗСБ широко используется при моделировании диффузии атомов в неупорядоченных средах, а ЗСУ - при исследовании процессов переноса электронных возбуждений и прыжковой проводимости по локализованным примесным центрам в полупроводниках и органических соединениях. Если частота прыжка имеет пороговый вид, то первая задача приводит к проблеме протекания по связям, а вторая - к протеканию по узлам. ЗСБ подробно исследована в пространстве произвольной размерности $d$ с использованием метода когерентного потенциала (МКП) [4]. Этот метод дает разумные результаты при произвольном распределении случайных барьеров (в отсутствие корреляций), хорошо согласующиеся с результатами моделирования методом Монте-Карло [1]. Что касается ЗСУ, то в большинстве работ, посвященных данной проблеме, для решения этой задачи фактически рассматривалась ЗСБ. Например, в работах [2], [5] при исследовании прыжковой проводимости использовалось приближение диагональной функции Грина, что вместе с парным приближением приводит к ЗСБ. В работе [6] предложен метод “эффективной среды” для решения задачи протекания по узлам в пространстве путем замены ЗСУ на ЗСБ на некоторой эффективной решетке. Этот прием использовался и во многих других работах при рассмотрении прыжковой проводимости для малой концентрации примесей [7]. Причиной этого является то, что между этими моделями имеется много общего. Действительно, в решетке, имеющей случайные узлы, диффузия происходит по случайным связям, которые коррелируют друг с другом, если они имеют общий узел. Во многих случаях в статическом пределе такими корреляциями можно пренебречь, и мы приходим к ЗСБ. Вместе с тем хорошо известно, что эти модели могут иметь и существенно различные свойства. Например, порог протекания для ЗСБ определяется, главным образом, координационным числом решетки $z$ (при рассмотрении протекания по ближайшим соседям), а для ЗСУ - параметром упаковки этой решетки. При этом ЗСУ в известном смысле оказывается более общей. Именно, ЗСБ можно всегда свести к ЗСУ на решетке иной геометрии [8].

Целью настоящей работы является попытка разработать новый алгоритм решения ЗСУ, который использует МКП - наиболее эффективное одноузельное приближение, известное в настоящее время. При этом мы сформулируем общий диаграммный метод нахождения коэффициента диффузии для ЗСУ, отличный от ЗСБ. ЗСУ будет рассмотрена на примере модели диффузии в гиперрешетке замещения размерности $d$ с некоррелированным статическим беспорядком, характеризующимся наличием заблокированных узлов с концентрацией $(1-c)$. Предполагается, что диффузия происходит путем прыжков между ближайшими незаблокированными узлами с постоянной частотой переходов $W$.

\section{2. УРАВНЕНИЕ ДЛЯ ДИФФУЗИОННОЙ ФУНКЦИИ В ПРЕДСТАВЛЕНИИ ЧИСЕЛ ЗАПОЛНЕНИЯ}

Рассмотрим уравнение баланса в представлении чисел заполнения [9] для функции условной вероятности $P_{n / m}(t)$ - вероятности того, что в момент времени $t$ ча- 
стица окажется на узле $n$, если при $t=0$ она была локализована на узле $m$ :

$$
s P_{n / m}(s)=c_{n} \delta_{n m}+\sum_{n^{\prime}}\left(P_{n^{\prime} / m} c_{n}-P_{n / m} c_{n^{\prime}}\right) W_{n n^{\prime}} .
$$

Здесь $P_{n / m}(s)$ - лапласов образ функции $P_{n / m}(t), W_{n n^{\prime}}=W$ - частота перехода между ближайшими узлами $n$ и $n^{\prime}$, а $c_{n}$ - случайная величина, характеризующая распределение доступных (незаблокированных) узлов:

$$
c_{n}= \begin{cases}1, & \text { если узел } n \text { является доступным, } \\ 0 & \text { в противном случае. }\end{cases}
$$

Поскольку предполагается отсутствие корреляции в распределении случайных узлов, для средних от произведения $c_{n}$ имеем

$$
\left\langle c_{n}\right\rangle=c, \quad\left\langle c_{n} c_{n}\right\rangle=c, \quad\left\langle c_{n} c_{n^{\prime}}\right\rangle=c^{2}, \quad n \neq n^{\prime},
$$

и т.д. Уравнение (1) удобно представить в матричном представлении, используя формализм Дирака:

$$
s \widehat{\mathbf{P}}=\widehat{\mathbf{C}}+\widehat{\mathbf{V}} \widehat{\mathbf{P}}
$$

где введены обозначения

$$
\begin{gathered}
\widehat{\mathbf{P}}=\sum_{n, n^{\prime}} P_{n / n^{\prime}}|n\rangle\left\langle n^{\prime}\right|, \\
\widehat{\mathbf{V}}=\sum_{n, n^{\prime}} c_{n} W_{n n^{\prime}}\left(|n\rangle-\left|n^{\prime}\right\rangle\right)\left\langle n^{\prime}\right|=\sum_{n} c_{n} \hat{\mathbf{v}}_{n}, \\
\widehat{\mathbf{C}}=\sum_{n} c_{n}|n\rangle\langle n|=\sum_{n} c_{n} \hat{\mathbf{p}}_{n} .
\end{gathered}
$$

Диффузионное уравнение (1) отличается от обычно используемых уравнений диффузии наличием случайного свободного члена, учитывающего невозможность размещения диффундирующей частицы в заблокированных узлах. Функция условной вероятности $P_{n / m}(s)$ удовлетворяет нормировочным условиям, следующим из закона сохранения полной вероятности:

$$
\sum_{n} s P_{n / m}(s)=c_{m}, \quad \sum_{m} s P_{n / m}(s)=c_{n} .
$$

Итерация уравнения (3) по свободному члену приводит к ряду

$$
\widehat{\mathbf{P}}=s^{-1} \sum_{n} c_{n} \hat{\mathbf{p}}_{n}+s^{-2} \sum_{n, n^{\prime}} c_{n} c_{n^{\prime}} \hat{\mathbf{v}}_{n} \hat{\mathbf{p}}_{n^{\prime}}+s^{-3} \sum_{n, n^{\prime}, n^{\prime \prime}} c_{n} c_{n^{\prime}} c_{n^{\prime \prime}} \hat{\mathbf{v}}_{n} \hat{\mathbf{v}}_{n^{\prime}} \hat{\mathbf{p}}_{n^{\prime \prime}}+\cdots
$$

Усредним каждый член этого ряда по случайному распределению $c_{n}$ согласно (2). Формально полученный ряд теории возмущений аналогичен рядам для решеточных моделей, используемым в теории неупорядоченных сплавов с диагональным беспорядком [10]. Для его анализа будем использовать метод кумулянтных разложений [11], [12]. При этом среднее от произведения любых случайных величин $c_{n}$ 
может быть разбито на сумму всевозможных произведений кумулянтных средних от этих величин [12]:

$$
\left\langle c_{n}\right\rangle=\left\langle c_{n}\right\rangle^{c}, \quad\left\langle c_{n} c_{n^{\prime}}\right\rangle=\left\langle c_{n}\right\rangle^{c}\left\langle c_{n^{\prime}}\right\rangle^{c}+\left\langle c_{n} c_{n^{\prime}}\right\rangle^{c}
$$

и т.д. Результат усреднения удобно представить в графическом виде, как показано на рис. 1. Светлому кружку сопоставляется множитель $\hat{\mathbf{p}}_{n}$, черному $-\hat{\mathbf{v}}_{n}$. По

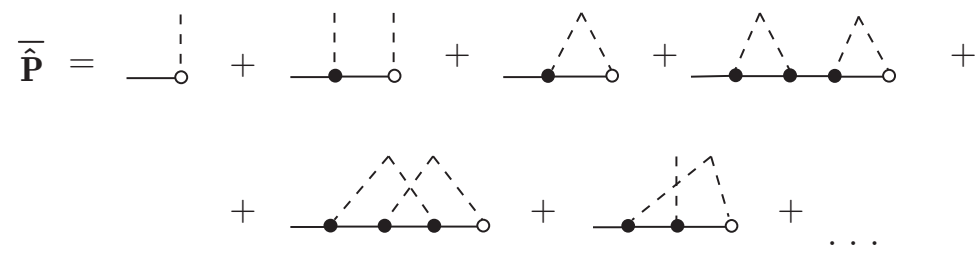

Рис. 1. Диаграммное представление конфигурационно-усредненой диффузионной функции $\widehat{\widehat{\mathbf{P}}}$.

номерам различных точек происходит суммирование. Всем горизонтальным сплошным линиям соответствует множитель $s^{-1}$, пучок штриховых (примесных) линий, соединяющий $m$ точек, обозначает кумулянтное среднее $Q_{m}(c)$ от $m$ множителей:

$$
Q_{m}(c)=\left\langle c_{1} c_{2} \ldots c_{m}\right\rangle^{c} .
$$

Из рис. 1 видно, что все диаграммы подразделяются на приводимые (которые можно разбить на две или более частей вертикальными сечениями, не пересекающими штриховые линии) и неприводимые. Обозначим через $\widehat{\mathbf{S}}$ сумму всех неприводимых диаграмм, которые содержат светлый кружок. Эти диаграммы изображены на рис. 2. Сумму всех остальных диаграмм обозначим через $\widehat{\mathbf{G}}$. Тогда

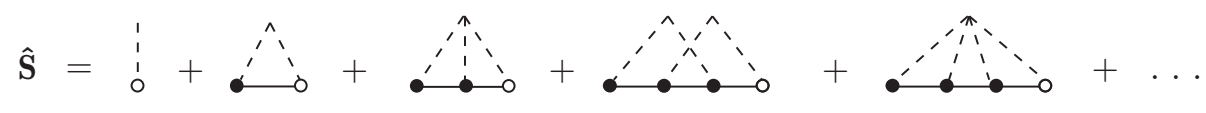

Рис. 2. Диаграммное представление функции $\widehat{\mathbf{S}}$.

конфигурационно-усредненная диффузионная функция $\widehat{\widehat{\mathbf{P}}}=\langle\widehat{\mathbf{P}}\rangle$ имеет вид

$$
\widehat{\widehat{P}}=\widehat{\mathbf{S}} \widehat{\mathbf{G}} .
$$

В свою очередь, класс диаграмм, относящихся к функции $\widehat{\mathbf{G}}$, может быть разбит на класс неприводимых диаграмм, сумму которых обозначим через $\widehat{\Pi}$ (массовый оператор), и класс приводимых диаграмм. Используя стандартную процедуру теории возмущений, легко получить уравнение Дайсона для функции $\widehat{\mathbf{G}}$ :

$$
s \widehat{\mathbf{G}}=\hat{\mathbf{I}}+\widehat{\mathbf{\Pi}} \widehat{\mathbf{G}},
$$

где $\hat{\mathbf{I}}$ - единичный оператор в пространстве состояний $|n\rangle$. На рис. 3 изображены 


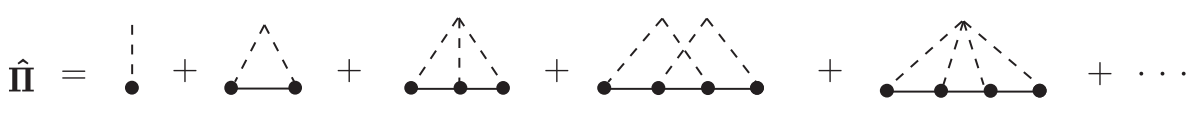

Рис. 3. Диаграммное представление массового оператора $\widehat{\boldsymbol{\Pi}}$.

диаграммы, представляющие массовый оператор $\widehat{\boldsymbol{\Pi}}$. Как видно из (8), функция $\widehat{\mathbf{G}}$ представляет собой функцию Грина. Эта функция описывает диффузию частицы, которая вначале помещается не на доступный узел, как в (1), а в произвольный узел решетки. Затем частица переходит на ближайшие незаблокированные узлы и далее диффундирует только по ним. Следовательно, статические коэффициенты диффузии, определяемые пропагаторами $\widehat{\boldsymbol{\Pi}}$ и $\widehat{\mathbf{G}}$, совпадают. Функция $\widehat{\mathbf{S}}$ учитывает корреляции, которые возникают из-за случайного распределения начальных условий в (3). Роль этой функции заключается в сокращении вклада диаграмм для диффузионной функции $\widehat{\widehat{\mathbf{P}}}$, которые начинаются на заблокированных узлах. Данная функция отсутствует в ЗСБ, поскольку в этой модели все узлы решетки изначально считаются доступными. Рассмотренная диаграммная техника была впервые предложена в работе [2].

\section{3. МЕТОД КОГЕРЕНТНОГО ПОТЕНЦИАЛА}

Точное суммирование диаграммных рядов для функций $\widehat{\boldsymbol{\Pi}}$ и $\widehat{\mathbf{S}}$ невозможно. Однако можно провести частичное суммирование этих рядов путем учета только одночастичных диаграмм, в которых все узлы совпадают. Это приближение соответствует МКП [10], [12]. Используя формальное сходство ряда для диффузионной функции (5) с рядом теории возмущений для функции Грина в модели сплава с диагональным беспорядком, легко получить уравнения для операторов $\widehat{\boldsymbol{\Pi}}$ и $\widehat{\mathbf{S}}$, которые соответствуют МКП для ЗСУ:

$$
\begin{array}{ll}
\widehat{\boldsymbol{\Pi}}=\sum_{n} \hat{\sigma}_{n}, & \hat{\sigma}_{n}=c \hat{\mathbf{v}}_{n}+\left(\hat{\mathbf{v}}_{n}-\hat{\sigma}_{n}\right) \widehat{\mathbf{G}} \hat{\sigma}_{n}, \\
\widehat{\mathbf{S}}=\sum_{n} \hat{\mathbf{s}}_{n}, & \hat{\mathbf{s}}_{n}=c \hat{\mathbf{p}}_{n}+\left(\hat{\mathbf{v}}_{n}-\hat{\sigma}_{n}\right) \widehat{\mathbf{G}} \hat{\mathbf{s}}_{n} .
\end{array}
$$

На языке диаграммной техники указанное приближение соответствует суммированию всех одночастичных диаграмм с заменой внутренних линий, соответствующих множителю $s^{-1}$, на линии, соответствующие полным функциям Грина $\widehat{\mathbf{G}}$. В результате получается самосогласованная система зацепляющихся уравнений для матричных элементов операторов $\hat{\sigma}_{n}$ и $\hat{\mathbf{s}}_{n}$. В модели диффузии по ближайшим узлам на гиперрешетке общее выражение для операторов $\hat{\sigma}_{n}$ и $\hat{\mathbf{s}}_{n}$ в МКП имеет вид

$$
\begin{aligned}
& \hat{\sigma}_{n}=W \sigma_{0}(s) \sum_{m} \Delta_{n, m}^{1}|m\rangle\left\langle m\left|+W \sigma_{1}(s) \sum_{m} \Delta_{n, m}^{1}\right| n\right\rangle\langle m|+ \\
& \quad+W \sigma_{2}(s) \sum_{m} \Delta_{n, l}^{1} \Delta_{n, m}^{1} \Delta_{l, m}^{2}|l\rangle\left\langle m\left|+W \sigma_{3}(s) \sum_{m} \Delta_{n, l}^{1} \Delta_{n, m}^{1} \Delta_{l, m}^{3}\right| l\right\rangle\langle m|, \\
& \hat{\mathbf{s}}_{n}=S_{0}|n\rangle\left\langle n\left|+S_{1} \sum_{m} \Delta_{n, m}^{1}\right| m\right\rangle\langle n|,
\end{aligned}
$$


где $\Delta_{n, m}^{\alpha}, \alpha=1,2,3,-$ функции обрезания, ограничивающие суммирование по узлам решетки, $\alpha$-й координационной сферой радиуса $R_{\alpha}$ :

$$
\Delta_{n, m}^{\alpha}= \begin{cases}1, & \text { если }\left|\vec{r}_{n}-\vec{r}_{m}\right|=R_{\alpha}, \\ 0 & \text { в противном случае. }\end{cases}
$$

Из выражений (10) следует, что величины $S_{0}, S_{1}$ и $\sigma_{i}, i=0,1,2,3$, полностью определяют диффузионную функцию $\widehat{\mathbf{P}}$. В свою очередь, они не являются независимыми и удовлетворяют соотношениям

$$
\begin{gathered}
\sigma_{0}+\sigma_{1}+2(d-1) \sigma_{2}+\sigma_{3}=0, \\
S_{0}+z S_{1}=c
\end{gathered}
$$

где $z=2 d$ - координационное число гиперрешетки размерности $d$. Следует отметить, что, в отличие от случая ЗСБ [4], выражение для оператора когерентного потенциала $\hat{\sigma}_{n}$ определяется тремя функциями $\sigma_{i}, i=1,2,3$, описывающими частоты переходов в эффективной гиперрешетке между узлами, расположенными на расстояниях трех ближайших координационных радиусов $R_{\alpha}, \alpha=1,2,3$. Подставляя (10) в (9), с учетом (11) находим выражения для массового оператора $\widehat{\boldsymbol{\Pi}}$ и оператора $\widehat{\mathbf{S}}$ в МКП:

$$
\begin{gathered}
\widehat{\mathbf{S}}=c \hat{\mathbf{I}}-z S_{1}\left(\hat{\mathbf{I}}-\hat{\boldsymbol{\varepsilon}}_{1}\right), \\
\widehat{\mathbf{\Pi}}=-z W\left[\sigma_{1}\left(\hat{\mathbf{I}}-\hat{\boldsymbol{\varepsilon}}_{1}\right)+2(d-1) \sigma_{2}\left(\hat{\mathbf{I}}-\hat{\boldsymbol{\varepsilon}}_{2}\right)+\sigma_{3}\left(\hat{\mathbf{I}}-\hat{\boldsymbol{\varepsilon}}_{3}\right)\right],
\end{gathered}
$$

где

$$
\hat{\boldsymbol{\varepsilon}}_{\alpha}=\frac{1}{2 d} \sum_{n, m} \Delta_{n, m}^{\alpha}|n\rangle\langle m|, \quad \alpha=1,2,3 .
$$

Переходя в $k$-представление, имеем

$$
\begin{gathered}
S(\vec{k}, s)=c-z S_{1}(s)\left(1-\varepsilon_{1}(\vec{k})\right), \\
\left.\Pi(\vec{k}, s)=-z W\left[\sigma_{1}(s)\left(1-\varepsilon_{1}(\vec{k})\right)\right]+2(d-1) \sigma_{1}(s)\left(1-\varepsilon_{2}(\vec{k})\right)+\sigma_{3}(s)\left(1-\varepsilon_{3}(\vec{k})\right)\right],
\end{gathered}
$$

где $\varepsilon_{\alpha}(\vec{k})$ - функция, определяющая закон дисперсии для $\alpha$-й координационной сферы:

$$
\varepsilon_{\alpha}(\vec{k})=\frac{1}{2 d} \sum_{m} \Delta_{n, m}^{\alpha} e^{i \vec{k}\left(\vec{r}_{n}-\vec{r}_{m}\right)} .
$$

Как видно из $(14)$, функция Грина $G(\vec{k}, s)$ при $k \rightarrow 0$ имеет диффузионный полюс:

$$
\begin{gathered}
\Pi(\vec{k}, s)=-W a^{2} \vec{k}^{2}\left[\sigma_{1}(s)+4(d-1) \sigma_{1}(s)+4 \sigma_{3}(s)\right], \\
S(\vec{k}, s)=c-S_{1}(s) a^{2} \vec{k}^{2} .
\end{gathered}
$$

При этом конфигурационно-усредненная диффузионная функция $\bar{P}(\vec{k}, s)$ в $k$-представлении равна

$$
\bar{P}(\vec{k}, s)=\frac{S(\vec{k}, s)}{s-\Pi(\vec{k}, s)} .
$$


Это позволяет выразить полный коэффициент диффузии рассматриваемой задачи через независимые функции $\sigma_{i}(s), i=1,2,3$, и $S_{1}(s)$ :

$$
D(s)=-\frac{s^{2}}{z} \lim _{\vec{k} \rightarrow 0} \frac{\hat{\triangle}_{\vec{k}} P(\vec{k}, s)}{c}=W a^{2}\left[s S_{1}(s)+\sigma_{1}(s)+4(d-1) \sigma_{1}(s)+4 \sigma_{3}(s)\right] .
$$

Получим самосогласованные уравнения для набора когерентных потенциалов $\sigma_{i}$ и функции $S_{1}$ в явном виде. Для этого подставим выражения (10) в уравнения (9) и вычислим матричные элементы соответствующих операторов между состояниями, определяемыми узлами решетки, лежащими на расстояниях трех ближайших координационных сфер. С учетом кубической симметрии решетки имеем

$$
\begin{aligned}
& S_{1}=-\sigma_{1}\left(1-\sigma_{1}\right) g_{1}, \\
& \sigma_{1}=c+\sigma_{1}\left(1-\sigma_{1}\right)\left(J_{3}+2(d-1) J_{2}-2 d J_{3}\right), \\
& \sigma_{2}=-b_{2}\left(1-\sigma_{1}\right)+\sigma_{2}\left(2 b_{2}-b_{1}-b_{3}\right), \\
& \sigma_{3}=-b_{3}\left(1-\sigma_{1}\right)+2(d-1) \sigma_{2}\left(b_{3}-b_{2}\right)+\sigma_{3}\left(b_{3}-b_{1}\right),
\end{aligned}
$$

где величины $b_{i}, i=1,2,3$, в свою очередь, удовлетворяют уравнениям

$$
\begin{aligned}
& b_{1}=\frac{(s / W) g_{0}-1}{z}, \\
& b_{2}=\left(J_{2}-J_{1}\right) \sigma_{1}+\left(2 J_{2}-J_{3}\right) \sigma_{2}, \\
& b_{3}=\left(J_{3}-J_{1}\right) \sigma_{1}+2(d-1)\left(J_{3}-J_{2}\right) \sigma_{2}+J_{3} \sigma_{3} .
\end{aligned}
$$

Функции $J_{\alpha}\left(s, \sigma_{1}, \sigma_{2}, \sigma_{3}\right), \alpha=1,2,3$, определяются с помощью решеточных сумм:

$$
\begin{aligned}
& J_{\alpha}(s)=W\left(\langle n|\widehat{G}| n\rangle-\Delta_{n, m}^{\alpha}\langle n|\widehat{G}| m\rangle\right)=W \sum_{\vec{k}} \frac{1-\varepsilon_{\alpha}(\vec{k})}{s-\Pi(\vec{k}, s)}, \\
& g_{1}(s)=W \Delta_{n, m}^{1}\langle n|\widehat{G}| m\rangle=W \sum_{\vec{k}} \frac{\varepsilon_{1}(\vec{k})}{s-\Pi(\vec{k}, s)}, \\
& g_{0}(s)=W\langle n|\widehat{G}| n\rangle=W \sum_{\vec{k}} \frac{1}{s-\Pi(\vec{k}, s)} .
\end{aligned}
$$

В общем случае получается замкнутая система трансцендентных уравнений для набора когерентных потенциалов $\sigma_{i}$, которую можно решить только численными методами. Для того чтобы проанализировать характер получаемых решений, найдем асимптотику этих выражений в пределе $c \rightarrow 1$. Известно, что в МКП учитываются все члены разложения для массового оператора $\widehat{\boldsymbol{\Pi}}$ и соответственно оператора $\widehat{\mathbf{S}}$ порядка $O(c(1-c))$. Тогда, удерживая в уравнениях (18) и (19) члены порядка $O(1-c)$, можно получить точную асимптотику коэффициента диффузии $D(s)$ при $c \rightarrow 1$ для ЗСУ. В результате имеем

$$
\frac{D(s)}{W a^{2}}=1-(1-c)\left[\frac{1+\bar{J}_{3}(s)}{1-\bar{J}_{3}(s)}+B(s)+s \bar{g}_{1}(s)\right]+o(1-c),
$$


где

$$
\begin{aligned}
B(s) & =\bar{J}_{3}(s)+2(d-1) \bar{J}_{2}(s)-2 d \bar{J}_{1}(s), \\
\bar{J}_{\alpha}(s) & =W \sum_{\vec{k}} \frac{1-\varepsilon_{\alpha}(\vec{k})}{s+z W\left(1-\varepsilon_{1}(\vec{k})\right)}, \\
\bar{g}_{1}(s) & =W \sum_{\vec{k}} \frac{\varepsilon_{1}(\vec{k})}{s+z W\left(1-\varepsilon_{1}(\vec{k})\right)} .
\end{aligned}
$$

Численный анализ показывает, что величиной $B(s)$ в выражении (21) можно пренебречь при $|s / W| \ll 1 \quad\left(B(0) \leqslant 10^{-5}\right.$ для $\left.d=2,3\right)$. В этом случае в статическом пределе $(s \rightarrow 0)$ коэффициент диффузии удобно представить в виде

$$
D(c)=\frac{1+\bar{J}_{3}}{1-\bar{J}_{3}}\left(c-c_{\mathrm{p}}\right),
$$

где $c_{\mathrm{p}}=2 \bar{J}_{3} /\left(1+\bar{J}_{3}\right)$.

Найденное выражение (22) совпадает с результатом работы [13], где рассматривалась задача диффузии меченого атома в мультикомпонентном сплаве, состоящем из нескольких сортов атомов, диффундирующих с различными скоростями по вакансионному механизму (динамический беспорядок). В предельном случае, когда все атомы с концентрацией $(1-c)$ становятся неподвижными, а меченый атом диффундирует по вакансиям, было получено выражение $(22)$. Величина $\bar{J}_{3}$ совпадает с известным выражением для среднего косинуса угла между направлениями последовательных прыжков вакансий при их диффузии в идеальной решетке [14]. Предполагая, что линейная зависимость (22) для коэффициента диффузии $D(c)$ имеет место для произвольной концентрации $c$, получаем порог протекания при концентрации доступных узлов, равной $c_{\mathrm{p}}[13]$.

\section{4. ОБСУЖДЕНИЕ ПОЛУЧЕННЫХ РЕЗУЛЬТАТОВ}

Следует отметить, что близкая к линейной зависимость коэффициента диффузии от концентрации с хорошей точностью подтверждается численными расчетами методом Монте-Карло практически для всех решеток как для ЗСУ, так и для ЗСБ. Лишь в непосредственной близости от порога протекания имеет место значительное отклонение от линейного закона, приводящее к различию реального порога протекания и порога протекания, получаемого с помощью линейной зависимости $(22)$.

Для того чтобы проанализировать поведение статического коэффициента диффузии $D(c)$ в МКП при произвольной концентрации доступных узлов $c$, было выполнено численное интегрирование системы уравнений (18)-(20). Результаты расчетов сравнивались с результатами, полученными с помощью моделирования методом Монте-Карло для трехмерной кубической решетки. Результаты расчетов представлены на рис. 4. Из рисунка видно, что кривая, полученная с использованием МКП, значительно отклоняется от кривой, полученной методом Монте-Карло при концентрации доступных узлов $c \leqslant 0.6$. Она обрывается в точке $c \approx 0.54$, где исчезает сходимость численной процедуры нахождения решения уравнений (18), (19). Эта 


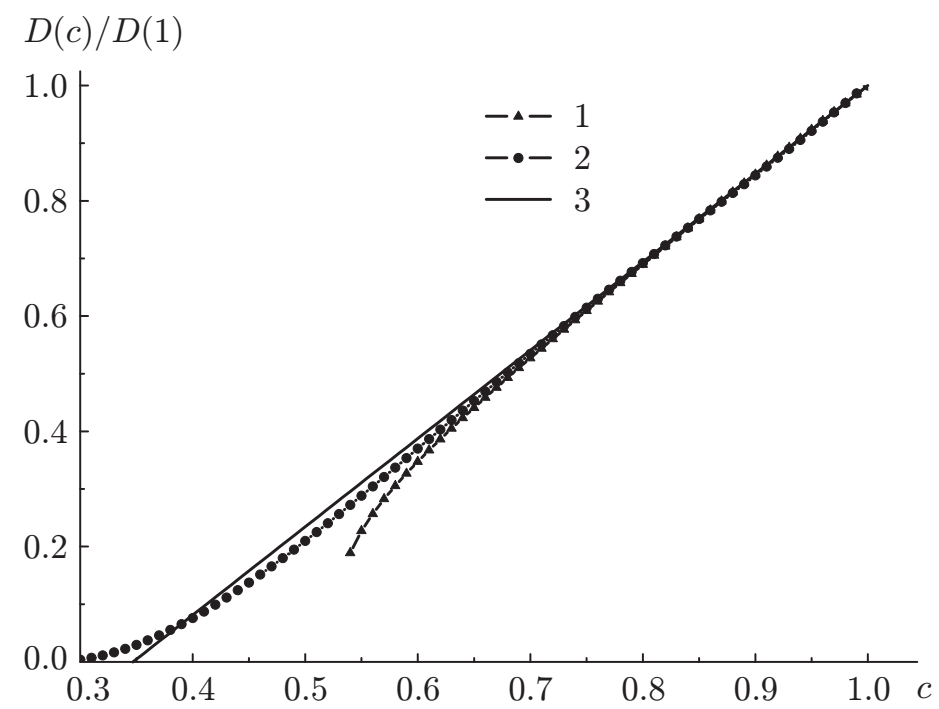

Рис. 4. Зависимость статического коэффициента диффузии от концентрации доступных узлов: 1 - МКП, 2 - метод Монте-Карло, 3 - линейная зависимость (22).

точка соответствует пределу сходимости ряда для массового оператора в приближении МКП. При $c>0.6$ обе кривые практически совпадают с линейной зависимостью вида (22). При увеличении размерности пространства область сходимости для массового оператора непрерывно увеличивается, а предел сходимости стремится к порогу протекания. Таким образом, можно заключить, что МКП достаточно хорошо описывает модель диффузии для ЗСУ в широком диапазоне изменения концентрации доступных узлов как для статического, так и для динамического режима. Однако при подходе к порогу протекания МКМ оказывается несостоятельным. Причиной этого является необходимость учета следующих членов разложения для диффузионной функции по концентрации доступных узлов вблизи порога протекания. Это приводит к выходу за рамки одноузельного приближения, что ведет к чрезвычайному усложнению диаграммных рядов и не позволяет получить замкнутые выражения для диффузионной функции. Использование результатов ЗСБ для описания диффузии в ЗСУ также имеет свои ограничения. Несмотря на то что статический коэффициент диффузии, полученный в рамках этой модели с помощью МКП, достаточно хорошо согласуется с линейной зависимостью вида (22) (в этой модели порог протекания равен $c_{\mathrm{p}}=1 / d$ и не зависит от типа решетки), это приближение оказывается некорректным для описания динамических свойств ЗСУ. Это является следствием того, что в ЗСБ не учитываются корреляции в распределении случайных связей, характерных для ЗСУ. Поскольку на малых и средних временах такие корреляции вносят заметный вклад в диффузионную функцию, аппроксимация ЗСУ с помощью ЗСБ в этом случае оказывается некорректной. 
Благодарности. Автор признателен Н. П. Лазареву за помощь при моделировании диффузии методом Монте-Карло.

\section{Список литературы}

[1] J. W. Hause, K. W. Kehr, Phys. Rep., 150 (1987), 263.

[2] В. В. Брыксин, Г. Ю. Яшин, ФTТ, 25 (1982), 3025.

[3] Н. П. Лазарев, М. П. Фатеев, ТМФ, 89:3 (1991), 465.

[4] I. Webman, J. Klafter, Phys. Rev. B, 26 (1982), 5958.

[5] R. F. Loring, H. C. Andersen, M. D. Fayer, J. Chem. Phys., 80 (1984), 5731.

[6] T. Odagaki, M. Lax, Phys. Rev. B, 36 (1987), 3851.

[7] В. В. Брыксин, ФTT, 26 (1984), 1362.

[8] D. Stauffer, A. Aharony, Introduction to Percolation Theory, Taylor and Francis, London, Washington DC, 1994.

[9] Ф. С. Джепаров, А. А. Лундин, ЖЭТФ, 75 (1978), 1017.

[10] R. J. Elliott, J. A. Krumhansl, P. L. Leath, Rev. Mod. Phys., 46:3 (1974), 465.

[11] R. Kubo, J. Phys. Soc. Japan, 17 (1962), 1100.

[12] F. Yonezawa, T. Matsubara, Progr. Theor. Phys. Suppl., 53 (1973), 1.

[13] R. A. Tahir-Kheli, Phys. Rev. B, 28 (1983), 3049.

[14] J. R. Manning, Phys. Rev. B, 4 (1971), 1111. 